\title{
Theoretical Research on Full Attitude Determination Using Geomagnetic Gradient Tensor
}

\author{
Yu Huang, Lihua Wu and Dequan Li
}

\author{
(Key Lab of In-fibre Integrated Optics, Education Ministry of China, Harbin \\ Engineering University, China) \\ (E-mail: huangyu@hrbeu.edu.cn)
}

To solve the problem of attitude determination using magnetometers independently and uniquely, which is important for underwater vehicles, a type of full attitude determination method based on geomagnetic gradient tensor is proposed in this paper. In this method, a group of non-linear equations concerning geomagnetic gradient tensors is established, where a quaternion is selected to calculate three attitude angles of an underwater vehicle. The optimal quaternion is estimated using Newton Down-hill to optimise the object function. The detailed steps of the full attitude determination based on geomagnetic gradient tensor are given, and the effects of the initial angle error and the sensor noise on the attitude determination are investigated. Simulations show that the algorithm can identify precisely and quickly the attitudes even in the presence of larger initial angle error and sensor noise, which proves the attitude determination algorithm.

\section{KEYWORDS \\ 1. Geomagnetic gradient tensor. 2. Attitude determination. 3. Underwater navigation.} 4. Newton down-hill method. 5. Quaternion.

Submitted: 12 September 2014. Accepted: 7 March 2015. First published online: 17 April 2015.

1. INTRODUCTION. High-precision attitude measurement and estimation is one of the most important problems in modern control, navigation and guidance systems. The performance of attitude determination systems with a gyroscope deteriorates over time due to the gyroscope drift, whose angle error can reach several degrees and exerts a negative effect on the accurate launch of submarine weapons. To carry out the realtime control of an underwater vehicle and high-precision guidance, it is necessary to guarantee high attitude determination precision.

Recently, the attitude determination methods based on different sensors have been put forward and widely applied in satellite navigation (Psiaki, 2004; Gebre-Egziaber et al., 2004; Lee et al., 2008). However, underwater GPS attitude determination technology does not allow for a high degree of autonomy and concealment as an 
underwater navigation system, and the underwater environment, leads to the abnormal reception of GPS signals (Psiaki, 2004; Gebre et al., 2004).

The precision and resolution of attitude determination using optical sensors decrease with the increasing distance of the target. On the other hand, optical sensor noise and light blocking may result in failure of attitude determination. Therefore, attitude determination based on optical measurement can not be used with reliability underwater (Lee et al., 2008; Liu et al., 2012; Kussat et al., 2005; Mukandan and Ramakrishnan, 1995). Expensive star sensor attitude measurement methods with the highest recognised precision are mainly applied to spacecraft, satellites and so on. These can independently determine attitude and need to be combined with an inertial navigation system to determine the relative attitude to the inertial space (Huang et al., 1993; Zhang et al., 2005; Jafarizadeh and Jamalipour, 2011; Kawano et al., 2002; Liebe, 2002). The attitude determination based on Earth's gravity and magnetic observation using a three-axis accelerometer and a three-axis magnetometer calculates the tilt angle according to the gravity vector and the heading angle in combination with the geomagnetic vector (Caruso, 2014). However, small tilt angle calculation error will lead to large head angle inaccuracy ( $\mathrm{Zhu}$ and $\mathrm{Xu}, 2006$ ).

From the above analysis, it is apparent that there are less effective attitude determination methods available that allow a craft navigating underwater to remain concealed. Using the geomagnetic field to determine an underwater vehicle's attitude is a good choice. However, it is impossible for a single vector magnetometer to independently determine attitude because the geomagnetic vector fails to provide three-axis independent information related to attitude, so a type of full attitude determination approach based on Earth's magnetic gradient tensor measurement is proposed. This method has the advantage of independence and can be applied in underwater navigation to safeguard underwater vehicles. Of course, it can also be used for the attitude determination of other craft. The magnetic gradient tensor measuring device is composed of four three-axis fluxgate magnetometers, which also has the potential advantage of low cost.

2. GEOMAGNETIC GRADIENT TENSOR AND ITS MEASUREMENT. The geomagnetic gradient tensor $G$ is the spatial variation rate of the geomagnetic vector along three directions in a Cartesian coordinate system, namely

$$
G=\left[\begin{array}{lll}
\frac{\partial H_{x}}{\partial x} & \frac{\partial H_{x}}{\partial y} & \frac{\partial H_{x}}{\partial z} \\
\frac{\partial H_{y}}{\partial x} & \frac{\partial H_{y}}{\partial y} & \frac{\partial H_{y}}{\partial z} \\
\frac{\partial H_{z}}{\partial x} & \frac{\partial H_{z}}{\partial y} & \frac{\partial H_{z}}{\partial z}
\end{array}\right]=\left[\begin{array}{lll}
g_{x x} & g_{x y} & g_{x z} \\
g_{y x} & g_{y y} & g_{y z} \\
g_{z x} & g_{z y} & g_{z z}
\end{array}\right]
$$

where, $H_{x}, H_{y}$ and $H_{z}$ are the three components in $x, y$ and $z$ directions, respectively. We can obtain $g_{x x}+g_{y y}+g_{z z}=0, g_{x y}=g_{y x}, g_{x z}=g_{z x}, g_{y z}=g_{z y}$ because the divergence and rotation of the geomagnetic field are all zero. Therefore, the magnetic gradient tensor is a $3 \times 3$ symmetrical matrix whose trace is zero and there exist five independent components selected as $g_{x x}, g_{y y}, g_{x y}, g_{y z}$ and $g_{z x}$ respectively.

To measure the geomagnetic gradient tensor accurately, a type of sensor configuration scheme called a ten single-axis configuration is adopted and shown in 


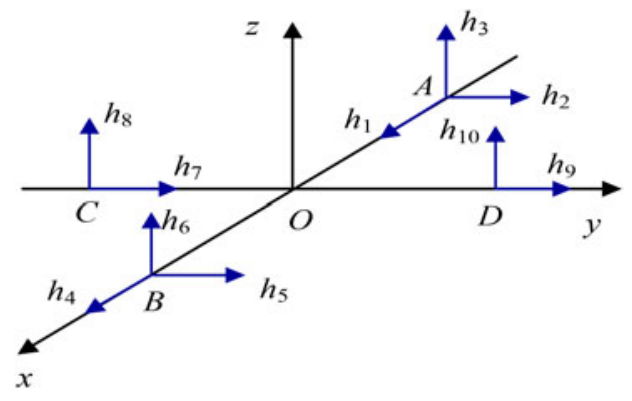

Figure 1. Diagram of geomagnetic gradient tensor measurement configuration.

Figure 1, where the arrows represent the sensitive axes of sensors. Two three-axis magnetometers are located at position $A$ to measure the magnetic field denoted as $\left(h_{1}, h_{2}\right.$, $\left.h_{3}\right)$ and $B$ to measure the magnetic field denoted as $\left(h_{4}, h_{5}, h_{6}\right)$, respectively. Similarly, the magnetic fields $\left(h_{7}, h_{8}\right)$ and $\left(h_{9}, h_{10}\right)$ are measured by two two-axis magnetometers located respectively at positions $C$ and $D$. The baseline lengths of gradient measurement in the directions $x$ and $y$ are respectively $l_{x}$ and $l_{y}$. Then, the geomagnetic gradient can be given by

$$
\left\{\begin{array}{l}
g_{x x}=\frac{h_{4}-h_{1}}{l_{x}}, g_{y y}=\frac{h_{9}-h_{7}}{l_{y}}, g_{y x}=\frac{h_{5}-h_{2}}{l_{x}} \\
g_{z y}=\frac{h_{10}-h_{8}}{l_{y}}, g_{z x}=\frac{h_{6}-h_{3}}{l_{x}}
\end{array}\right.
$$

The local perturbations or anomalies from rocks, terrain, man-made objects, etc can be viewed as the components of Earth's magnetic gradient tensor, and these tensor measurements can be accurately surveyed by a precise gradient tensor system through calibration, in which resolution can reach $0 \cdot 52 \mathrm{nT} / \mathrm{m}$ (Sui et al., 2014).

\section{ATTITUDE MEASUREMENT PRINCIPLE BASED ON MAGNETIC} GRADIENT TENSOR. As shown in Figure 2, the navigation coordinate system is represented by $o x_{n} y_{n} z_{n}$, whose three axes are respectively east, north and up, and $o x_{b} y_{b} z_{b}$ is the vehicle coordinate system. The three angles $\psi, \theta$ and $\gamma$ are the heading, pitch and roll angles. Consequently, the relationship between the navigation coordinate and vehicle coordinate can be described as follows: firstly, $o x_{1} y_{1} z_{1}$ is obtained by rotating $o x_{n} y_{n} z_{n}$ with $\psi$ around $-o z_{n}$; secondly, $O x_{2} y_{2} z_{2}$ is obtained by rotating $O x_{1} y_{1} z_{1}$ with $\theta$ around $o x_{1}$; finally, $O x_{b} y_{b} z_{b}$ can be given by rotating $O x_{2} y_{2} z_{2}$ around $o y_{2}$.

The transformation matrix $C_{n}^{b}$ from the navigation coordinate system to the vehicle coordinate system can be written as

$$
C_{n}^{b}=\left[\begin{array}{ccc}
\cos \gamma \cos \psi+\sin \gamma \sin \psi \sin \theta & -\cos \gamma \sin \psi+\sin \gamma \cos \psi \sin \theta & -\sin \gamma \cos \theta \\
\sin \psi \cos \theta & \cos \psi \cos \theta & \sin \theta \\
\sin \gamma \cos \psi-\cos \gamma \sin \psi \sin \theta & -\sin \gamma \sin \psi-\cos \gamma \cos \psi \sin \theta & \cos \gamma \cos \theta
\end{array}\right]
$$




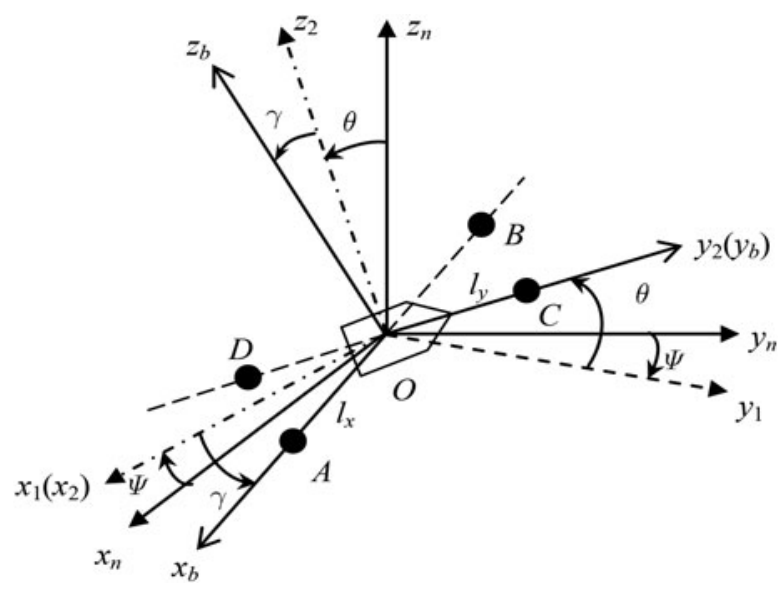

Figure 2. Schematic diagram for attitude measurement principle.

The quantities $G^{n}$ and $G^{b}$ show the magnetic gradient tensors in the navigation coordinate system and vehicle coordinate system, respectively. Thus,

$$
G^{b}=C_{n}^{b} G^{n}\left(C_{n}^{b}\right)^{T}
$$

where, $T$ expresses the matrix transpose. The quantity $G^{b}$ can be obtained by a magnetic gradient measurement device because it is generally strapped on the vehicle. On the other hand, $G^{n}$ can be measured in advance and stored in the navigation computer as the navigation geophysical database. From Equation (4), we can obtain five independent equations for the attitude angles $(\psi, \theta, \gamma)$. To avoid trigonometric function operation of the optimal estimation, the quaternion $Q=q_{0}+q_{1} i_{0}+q_{2} j_{0}+q_{3} k_{0}$ is introduced to determine the transform matrix, namely

$$
C_{b}^{n}=\left(C_{b}^{n}\right)^{T}=\left[\begin{array}{ccc}
q_{0}^{2}+q_{1}^{2}-q_{2}^{2}-q_{3}^{2} & 2\left(q_{1} q_{2}-q_{0} q_{3}\right) & 2\left(q_{1} q_{3}+q_{0} q_{2}\right) \\
2\left(q_{1} q_{2}+q_{0} q_{3}\right) & q_{0}^{2}-q_{1}^{2}+q_{2}^{2}-q_{3}^{2} & 2\left(q_{2} q_{3}-q_{0} q_{1}\right) \\
2\left(q_{1} q_{3}-q_{0} q_{2}\right) & 2\left(q_{2} q_{3}+q_{0} q_{1}\right) & q_{0}^{2}-q_{1}^{2}-q_{2}^{2}+q_{3}^{2}
\end{array}\right]
$$

From Equations (4) and (5), we can deduce the following five independent equations with regard to $q_{0}, q_{1}, q_{2}$ and $q_{3}$

$$
\begin{aligned}
g_{x x}^{b}= & g_{x x}^{n}\left(q_{0}^{2}+q_{1}^{2}-q_{2}^{2}-q_{3}^{2}\right)^{2}+4 g_{y y}^{n}\left(q_{1} q_{2}+q_{0} q_{3}\right)^{2} \\
& +4 g_{x y}^{n}\left(q_{0}^{2}+q_{1}^{2}-q_{2}^{2}-q_{3}^{2}\right)\left(q_{1} q_{2}+q_{0} q_{3}\right) \\
& +8 g_{z y}^{n}\left(q_{1} q_{2}+q_{0} q_{3}\right)\left(q_{1} q_{3}-q_{0} q_{2}\right) \\
& +4 g_{x z}^{n}\left(q_{0}^{2}+q_{1}^{2}-q_{2}^{2}-q_{3}^{2}\right)\left(q_{1} q_{3}-q_{0} q_{2}\right) \\
& -4\left(g_{x x}^{n}+g_{y y}^{n}\right)\left(q_{1} q_{3}-q_{0} q_{2}\right)^{2}
\end{aligned}
$$




$$
\begin{aligned}
& g_{y y}^{b}=4 g_{x x}^{n}\left(q_{1} q_{2}-q_{0} q_{3}\right)^{2}+g_{y y}^{n}\left(q_{0}^{2}-q_{1}^{2}+q_{2}^{2}-q_{3}^{2}\right)^{2} \\
& +4 g_{y x}^{n}\left(q_{0}^{2}-q_{1}^{2}+q_{2}^{2}-q_{3}^{2}\right)\left(q_{1} q_{2}-q_{0} q_{3}\right) \\
& +4 g_{z y}^{n}\left(q_{0}^{2}-q_{1}^{2}+q_{2}^{2}-q_{3}^{2}\right)\left(q_{2} q_{3}+q_{0} q_{1}\right) \\
& +8 g_{z x}^{n}\left(q_{1} q_{2}-q_{0} q_{3}\right)\left(q_{2} q_{3}+q_{0} q_{1}\right) \\
& -4\left(g_{x x}^{n}+g_{y y}^{n}\right)\left(q_{2} q_{3}-q_{0} q_{1}\right)^{2} \\
& g_{y x}^{b}=2 g_{x x}^{n}\left(q_{0}^{2}+q_{1}^{2}-q_{2}^{2}-q_{3}^{2}\right)\left(q_{1} q_{2}-q_{0} q_{3}\right)+2 g_{y y}^{n}\left(q_{0}^{2}-q_{1}^{2}+q_{2}^{2}-q_{3}^{2}\right)\left(q_{1} q_{2}+q_{0} q_{3}\right) \\
& +g_{y x}^{n}\left[\left(q_{0}^{2}+q_{1}^{2}-q_{2}^{2}-q_{3}^{2}\right)\left(q_{0}^{2}-q_{1}^{2}+q_{2}^{2}-q_{3}^{2}\right)+4\left(q_{1} q_{2}-q_{0} q_{3}\right)\left(q_{1} q_{2}+q_{0} q_{3}\right)\right] \\
& +2 g_{z y}^{n}\left[2\left(q_{1} q_{2}+q_{0} q_{3}\right)\left(q_{2} q_{3}+q_{0} q_{1}\right)+\left(q_{0}^{2}-q_{1}^{2}+q_{2}^{2}-q_{3}^{2}\right)\left(q_{1} q_{3}-q_{0} q_{2}\right)\right] \\
& +2 g_{z x}^{n}\left[2\left(q_{0}^{2}+q_{1}^{2}-q_{2}^{2}-q_{3}^{2}\right)\left(q_{2} q_{3}+q_{0} q_{1}\right)+2\left(q_{1} q_{3}-q_{0} q_{2}\right)\left(q_{1} q_{2}-q_{0} q_{3}\right)\right] \\
& -4\left(g_{x x}^{n}+g_{y y}^{n}\right)\left(q_{1} q_{3}-q_{0} q_{2}\right)\left(q_{2} q_{3}+q_{0} q_{1}\right) \\
& g_{z y}^{b}=4 g_{x x}^{n}\left(q_{1} q_{2}-q_{0} q_{3}\right)\left(q_{1} q_{3}+q_{0} q_{2}\right)+2 g_{y y}^{n}\left(q_{0}^{2}-q_{1}^{2}+q_{2}^{2}-q_{3}^{2}\right)\left(q_{2} q_{3}+q_{0} q_{1}\right) \\
& +2 g_{y x}^{n}\left[2\left(q_{1} q_{2}-q_{0} q_{3}\right)\left(q_{2} q_{3}-q_{0} q_{1}\right)+\left(q_{0}^{2}-q_{1}^{2}+q_{2}^{2}-q_{3}^{2}\right)\left(q_{1} q_{3}+q_{0} q_{2}\right)\right] \\
& +g_{z y}^{n}\left[\left(q_{0}^{2}-q_{1}^{2}+q_{2}^{2}-q_{3}^{2}\right)\left(q_{0}^{2}-q_{1}^{2}-q_{2}^{2}+q_{3}^{2}\right)+4\left(q_{0} q_{1}+q_{2} q_{3}\right)\left(q_{2} q_{3}-q_{0} q_{1}\right)\right] \\
& +2 g_{z x}^{n}\left[2\left(q_{0}^{2}-q_{1}^{2}-q_{2}^{2}+q_{3}^{2}\right)\left(q_{1} q_{2}-q_{0} q_{3}\right)+2\left(q_{2} q_{3}+q_{0} q_{1}\right)\left(q_{1} q_{3}-q_{0} q_{2}\right)\right] \\
& -2\left(g_{x x}^{n}+g_{y y}^{n}\right)\left(q_{0}^{2}-q_{1}^{2}-q_{2}^{2}+q_{3}^{2}\right)\left(q_{2} q_{3}+q_{0} q_{1}\right) \\
& g_{z x}^{b}=2 g_{x x}^{n}\left(q_{0}^{2}+q_{1}^{2}-q_{2}^{2}-q_{3}^{2}\right)\left(q_{1} q_{3}+q_{0} q_{2}\right)+4 g_{y y}^{n}\left(q_{1} q_{2}+q_{0} q_{3}\right)\left(q_{2} q_{3}-q_{0} q_{1}\right) \\
& +2 g_{y x}^{n}\left[2\left(q_{1} q_{2}+q_{0} q_{3}\right)\left(q_{1} q_{3}+q_{0} q_{2}\right)+\left(q_{0}^{2}+q_{1}^{2}-q_{2}^{2}-q_{3}^{2}\right)\left(q_{2} q_{3}+q_{0} q_{1}\right)\right] \\
& +2 g_{z y}^{n}\left[\left(q_{0}^{2}-q_{1}^{2}-q_{2}^{2}+q_{3}^{2}\right)\left(q_{1} q_{2}+q_{0} q_{3}\right)+2\left(q_{1} q_{3}-q_{0} q_{2}\right)\left(q_{2} q_{3}-q_{0} q_{1}\right)\right] \\
& +g_{z x}^{n}\left[2\left(q_{0}^{2}+q_{1}^{2}-q_{2}^{2}-q_{3}^{2}\right)\left(q_{0}^{2}-q_{1}^{2}-q_{2}^{2}+q_{3}^{2}\right)+4\left(q_{1} q_{3}+q_{0} q_{2}\right)\left(q_{1} q_{3}-q_{0} q_{2}\right)\right] \\
& -2\left(g_{x x}^{n}+g_{y y}^{n}\right)\left(q_{0}^{2}-q_{1}^{2}-q_{2}^{2}+q_{3}^{2}\right)\left(q_{1} q_{3}-q_{0} q_{2}\right)
\end{aligned}
$$

In addition there still exists the following relation for the quaternion

$$
q_{0}^{2}+q_{1}^{2}+q_{2}^{2}+q_{3}^{2}=1
$$

The above six equations can be replaced by

$$
f_{i}\left(q_{0}, q_{1}, q_{2}, q_{3}\right)=0(i=1,2, \ldots, 6)
$$

Equation (12) is a group of nonlinear equations, which is traditionally solved using an iterative method, the Newton method, the steepest descent method or conjugate gradient method, etc. According to characteristics of the nonlinear equations shown as Equation (12), the Newton Downhill method is chosen, which is a fast and effective method for solving nonlinear equations. The iterative formula of Newton Downhill 
Table 1 . The truth table of heading angle.

\begin{tabular}{lll}
\hline$\hat{c}_{22}$ & $\hat{c}_{12}$ & $\hat{\psi}$ \\
\hline$\rightarrow 0$ & + & $90^{\circ}$ \\
$\rightarrow 0$ & - & $-90^{\circ}$ \\
+ & + & $\hat{\psi}_{\text {main }}$ \\
+ & - & $\hat{\psi}_{\text {main }}$ \\
- & + & $\hat{\psi}_{\text {main }}+180^{\circ}$ \\
- & - & $\hat{\psi}_{\text {main }}-180^{\circ}$ \\
\hline
\end{tabular}

Table 2. The truth table of roll angle.

\begin{tabular}{lll}
\hline$\hat{\gamma}_{\text {main }}$ & $\hat{c}_{33}$ & $\hat{\gamma}$ \\
\hline+ & + & $\hat{\gamma}_{\text {main }}$ \\
- & + & $\hat{\gamma}_{\text {main }}$ \\
+ & - & $\hat{\gamma}_{\text {main }}-180^{\circ}$ \\
- & - & $\hat{\gamma}_{\text {main }}+180^{\circ}$ \\
\hline
\end{tabular}

method is

$$
x^{n+1}=x^{n}-\omega\left(F^{\prime}\left(x^{n}\right)\right)^{-1} F\left(x^{n}\right)
$$

where $0<\omega \leq 1$. To guarantee convergence, $\omega$ generally meets

$$
\left\|F\left(x^{n+1}\right)\right\|<\left\|F\left(x^{n}\right)\right\|
$$

We adopt the successive halving method to determine $\omega$. To lighten the burden of calculation, partial derivatives in the Jacobian matrix are replaced by difference approximation.

The solutions $\hat{q}_{0}, \hat{q}_{1}, \hat{q}_{2}, \hat{q}_{3}$ of Equation (12) are given using the above iterative algorithm, and then matrix elements $\hat{c}_{i j}$ of $C_{b}^{n}$ are obtained $(i, j=1,2,3)$ through substituting $\hat{q}_{3}, \hat{q}_{1}, \hat{q}_{2}, \hat{q}_{3}$ into Equation (5). The following relations can be given using Equations (3) and (4),

$$
\left\{\begin{array}{l}
\hat{\gamma}_{\text {main }}=\arctan \left(-\frac{\hat{c}_{31}}{\hat{c}_{33}}\right) \\
\theta=\arcsin \left(\hat{c}_{32}\right) \\
\hat{\psi}_{\text {main }}=\arctan \left(\frac{\hat{c}_{12}}{\hat{c}_{22}}\right)
\end{array}\right.
$$

where, $\hat{\theta}$ is the estimated pitch angle; $\hat{\psi}_{\text {main }}$ and $\hat{\gamma}_{\text {main }}$ are respectively the main values of estimated heading and roll angles. The estimated heading and roll angles are decided by the rules shown in Tables 1 and 2, respectively.

The detailed steps for underwater full attitude determination based on geomagnetic gradient tensor measurement are described as follows.

Step 1: Calculate the five components of geomagnetic gradient tensor in the vehicle coordinate system according to the sensor output from the magnetic tensor measurement device and Equation (2); 


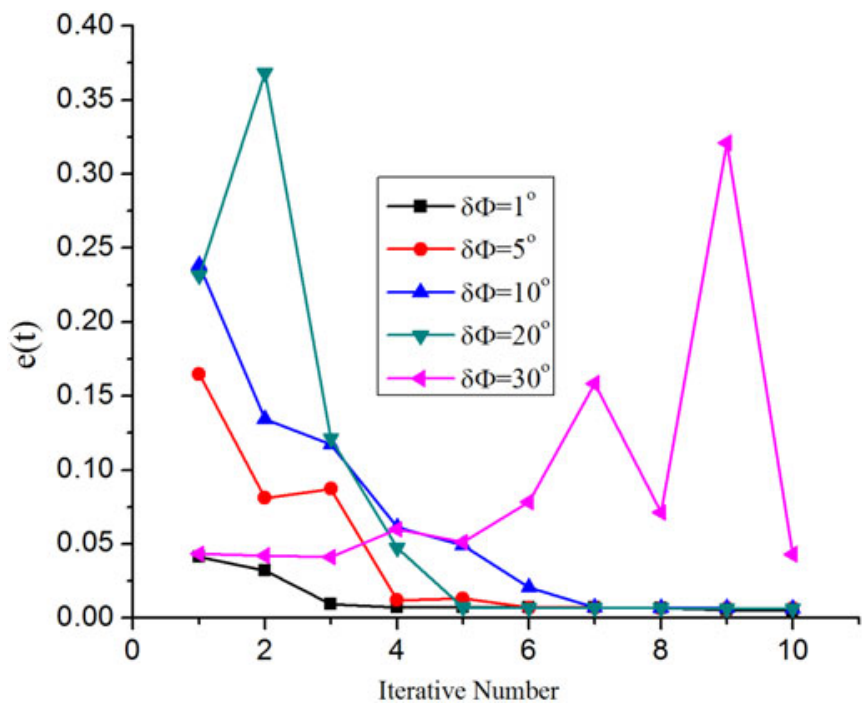

Figure 3. The convergence algorithm under different initial solution conditions.

Step 2: Extract the five components of magnetic gradient tensor in the navigation coordinate system from the geomagnetic navigation database according to the position indicated by the inertial navigation system;

Step 3: Solve the nonlinear Equation (12) and obtain $\hat{q}_{0}, \hat{q}_{1}, \hat{q}_{2}$ and $\hat{q}_{3}$ using the Newton Downhill method;

Step 4: Substitute $\hat{q}_{0}, \hat{q}_{1}, \hat{q}_{2}$ and $\hat{q}_{3}$ into Equation (5) to calculate $\hat{c}_{i j}$, and then calculate three attitude angles according to Equation (15) and Tables 1 and 2.

4. NUMERICAL EXPERIMENTS. We use a single magnetic dipole to produce a geomagnetic anomaly gradient, whose magnetic moments in three directions of the navigation coordinate system are $m_{x}=10^{9} \mathrm{~A} \cdot \mathrm{m}^{2}, m_{y}=2 \times 10^{8} \mathrm{~A} \cdot \mathrm{m}^{2}$ and $m_{z}=10^{8}$ $\mathrm{A} \cdot \mathrm{m}^{2}$, respectively. The spatial positions of the magnetic dipole relative to the magnetic gradient tensor measurement device are $x=100 \mathrm{~m}, y=50 \mathrm{~m}$ and $z=20 \mathrm{~m}$ in the navigation coordinate system, respectively. The baseline lengths of magnetic gradient measurement are both $1 \mathrm{~m}$, namely $l_{x}=l_{y}=1 \mathrm{~m}$. The iterative termination condition of the Newton Downhill algorithm is selected as $\varepsilon=\left\|x_{k+1}-x_{k}\right\| \leq 10^{-6}$.

Firstly, the influence of the initial solution on the algorithm accuracy is investigated. The noise of each magnetometer is assumed to be an independent Gaussian noise process with zero mean and variance of $\sigma=2 n T$.

Figure 3 shows the convergence of the Newton Downhill method for different initial solutions, where $\delta \Phi$ represents the difference of initial solutions deviating from the true solution. When the difference is very obvious, for example, $\delta \Phi=20^{\circ}$ and $\delta \Phi=30^{\circ}$, the error $e(t)$ will fluctuate markedly. But when $\delta \Phi=20^{\circ}$, the Newton Downhill method still shows convergence after a few iterations, which means that the error is less than the given minimum.

The Newton Downhill method generally shows good convergence through not more than 20 iteration steps when $\delta \Phi \leq 20^{\circ}$, which is proved by many simulations. This 


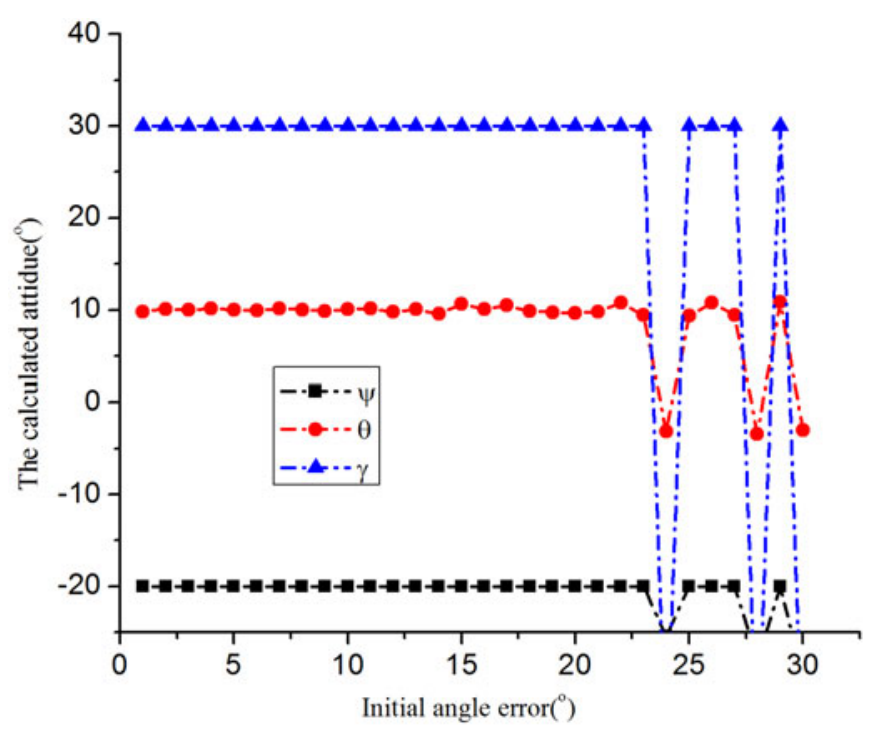

Figure 4. The estimated attitude angles for different initial solutions.

shows the full attitude determination algorithm costs less computing time and possesses potential application value.

Figure 4 gives the estimated attitude angles in the case of different initial angle errors. From Figure 4, we can see the full attitude determination algorithm is not sensitive to initial angle error, and can estimate effectively the attitude angles as long as initial angle error is less than $20^{\circ}$, where the estimation errors of $\theta, \psi$ and $\gamma$ are about $0 \cdot 47^{\circ}, 0 \cdot 15^{\circ}, 0 \cdot 12^{\circ}$, respectively. This further indicates that the full attitude determination algorithm possesses potential application value. The output attitude error of the inertial navigation system is less than $10^{\circ}$, which can be used as the initial attitude of the attitude determination algorithm. The error of the full attitude determination algorithm based on a geomagnetic gradient tensor is less than $0.5^{\circ}$, which remarkably improves the precision of the navigation system.

Next, the influence of the sensor noise on the algorithm accuracy is investigated with other invariable simulation parameters. The resolution and absolute precision of a good fluxgate magnetometer such as the RM100 are respectively $0 \cdot 1 \mathrm{nT}$ and $1 \mathrm{nT}$. The resolution of a superconducting vector magnetometer such as the $755 \mathrm{R}$ can reach $2 \times 10^{-3} \mathrm{nT}$. The resolution and relative accuracy of a laser optical pump helium vector magnetic sensor such as the P-2000 are better than $0 \cdot 01 \mathrm{nT}$ and $0 \cdot 1 \%$, respectively. Figure 5 shows the convergence of the algorithm for different sensor noise expressed by $\sigma$, which is lower than $10 \mathrm{nT}$.

From Figure 5, we can see that convergence of the algorithm is not obviously influenced by the sensor noise as long as it is less than $10 \mathrm{nT}$, when the maximum number of iterations is 18 . However, the convergence speed slows significantly when the noise is higher than $10 \mathrm{nT}$.

Figure 6 gives the estimated attitude angles in conditions of different magnetometer noise. When the noise is less than $10 \mathrm{nT}$, the estimation errors of $\theta, \psi$ and $\gamma$ are about $0 \cdot 35^{\circ}, 0 \cdot 11^{\circ}, 0 \cdot 13^{\circ}$, respectively. The noise of a low-cost three-axis fluxgate sensor is less 


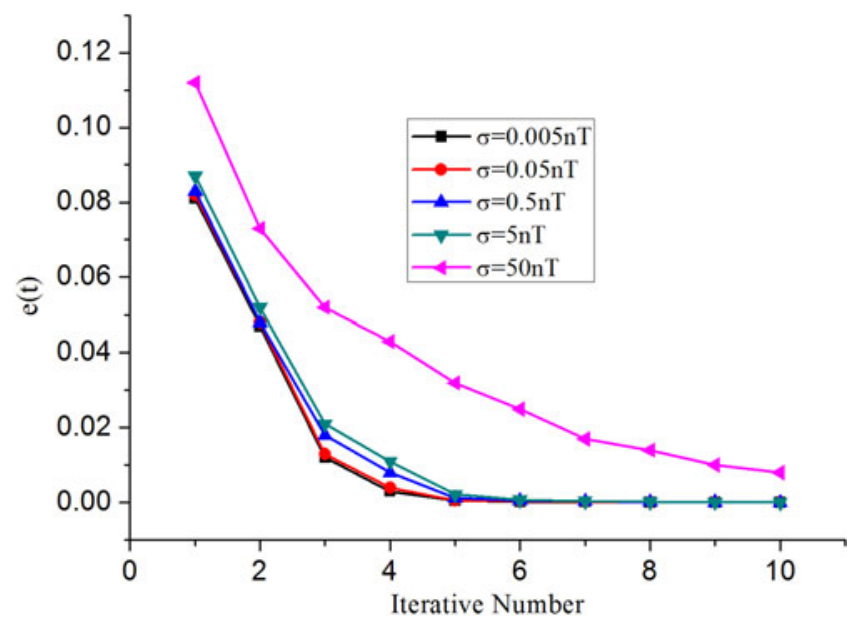

Figure 5. Algorithm convergence under different noise levels.

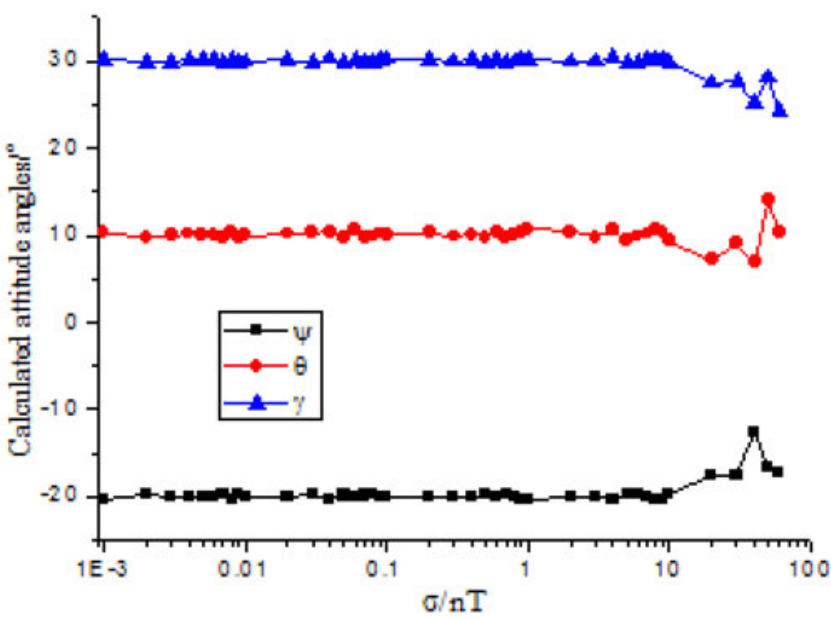

Figure 6. Attitude angle estimation under different noise levels.

than $10 \mathrm{nT}$, and therefore it is feasible for the algorithm to use low-cost three-axis fluxgate sensors to construct a geomagnetic gradient tensor measurement device.

During surveying, the device straps down the inertial platform with gyro of $0.01^{\mathrm{o}} / \mathrm{h}$ integrated with other sensors, and then its horizontal misalignment is better than $1^{\prime}$. The errors in platform attitude are viewed directly as attitude determination error, so its effect on attitude determination can be ignored. On the other hand, the errors in location during surveying will also lead to gradient tensor error, and the relationship between them in the surveying plane can be expressed as

$$
\Delta g_{i j}=\frac{\partial g_{i j}}{\partial x} \Delta x+\frac{\partial g_{i j}}{\partial y} \Delta y
$$


where, $i, j=x, y, z, \Delta g_{i j}$ is the gradient tensor error and $\Delta x$ and $\Delta y$ are the location errors. According the above simulations, the attitude determination precision is acceptable when the noise of fluxgate sensor is less than $10 \mathrm{nT}$. Correspondingly, the errors of gradient tensor are about $10 \mathrm{nT} / \mathrm{m}$. So, $\Delta g_{i j}$ should be less than $10 \mathrm{nT} / \mathrm{m}$ and the errors in location are determined by Equation (16).

5. CONCLUSIONS. Attitude measurement and estimation is one of the most important parts of underwater navigation and control. An underwater full attitude determination method based on geomagnetic gradient tensor measurement is proposed in this paper and its steps are given. The effects of the initial angle error and the sensor noise on the attitude determination are studied by simulation. The results show that the algorithm has the advantages of higher precision and faster convergence when the initial angle error is less than $20^{\circ}$ and the sensor noise is less than $10 \mathrm{nT}$. We can conclude that the algorithm possesses potential application value because an inertial navigation system can provide the initial angles, whose error is less than $10^{\circ}$, and low-cost fluxgate magnetometers can be used to measure geomagnetic gradient tensor.

\section{ACKNOWLEDGEMENT}

This work was supported by the National Nature Science Foundation of China under Grant Nos 61004130, Postdoctoral Science Foundation of China under Grant Nos 2012M511446, 2013T60348 and 2013M530145, the Fundamental Research Funds for the Central Universities, the 111 project (B13015), to the Harbin Engineering University, Natural Science Foundation of Heilongjiang Province of China under Grant Nos F201415.

\section{REFERENCES}

Caruso, M.J. (2014). Application of Magnetic Sensors for Low Cost Compass Systems. http://www.ssec.hon eywell.com/magnetic/datasheets/lowcost.pdf (Accesse 16 April 2014).

Gebre-Egziaber, D., Hayward, R.C., and Powell, J.D. (2004). Design of multi-sensor attitude determination systems. IEEE Transactions on Aerospace and Electronic Systems, 40(2), 627-649.

Huang, Y., Zhou, S., Wang, L., Fan, R. and Wu, H. (1993). The optical measurement of pose parameters of target spacecraft. Journal of Astronautics, 14(1), 97-103. (in Chinese)

Jafarizadeh, S. and Jamalipour, A. (2011). Fastest Distributed Consensus Problem on Fusion of Two Star Sensor Networks. IEEE Sensors Journal, 11(10), 2494-2505.

Kawano, H., Sato, Y., Mitani, K., Kanai, H. and Hama, K. (2002). New light shielding technique for shortening the baffle length of a star sensor. Proceedings of SPIE - The International Society for Optical Engineering, 4767:62-69.

Kussat, N.H., Chadwell, C.D. and Zimmerman, R. (2005). Absolute Positioning of an Autonomous Underwater Vehicle Using GPS and Acoustic Measurements. IEEE Journal of Oceanic Engineering, 30(1), 153-164.

Lee, H., Choi, Y., Bang, H. and Park, J. (2008). Kalman filtering for spacecraft attitude estimation by lowcost sensors. International Journal of Aeronautical and Space Sciences, 9(1), 147-161.

Liebe, C. (2002). Accuracy performance of Star Trackers a Tutorial. IEEE Transactions on Aerospace and Electronic Systems, 38(2), 687-599.

Liu, L., Wang, R. and Xiao, F. (2012). Topology Control Algorithm for Underwater Wireless Sensor Networks Using GPS-free Mobile Sensor Nodes. Journal of Network and Computer Applications, 35, 1953-1963.

Mukundan, R. and Ramakrishnan, K.R. (1995). A quaternion solution to the pose determination problem for rendezvous and docking simulations. Mathematics and Computers in Simulation, 39, 143-153. 
Psiaki, M.L. (2004). Global magnetometer based spacecraft attitude and rate estimation. Journal of Guidance, Control and Dynamics, 27(2), 240-250.

Qiang, J., Costas, M.S., Haralick, R.M. and Shapiro, L. (2000). A robust linear least-squares estimation of camera exterior orientation using multiple geometric features. ISPRS Journal of Photogrammetry and Remote Sensing, 55, 75-93.

Sui, S., Li, G., Wang, S. and Lin, J. (2014). Compact fluxgate magnetic full-tensor gradiometer with spherical feedback coil. Review of Scientific Instruments, 85(1), 014701.

Zhang, S., Cao, X. and Chen, X. (2005). Closed-form method for relative pose parameters of spacecraft based on optical measurement. Acta Aeronautica and Astronautica Sinica, 26(2), 214-218.

$\mathrm{Zhu}$, J. and Xu, S. (2006). UKF Algorithm for Satellite Attitude Estimation Based on the Magnetic Field Measurement. Journal of Astronautics, 27(6), 1401-1405. 